\title{
The sequence variation and functional differentiation of CRDs in a scallop multiple CRDs containing lectin
}

\author{
Mengmeng Huang ${ }^{a}$, Lingling Wang ${ }^{b}$, Huan Zhang ${ }^{c}$, Chuanyan Yang ${ }^{b}$, Rui Liu ${ }^{c}$, \\ Jiachao $\mathrm{Xu}^{\mathrm{c}}$, Zhihao Jia ${ }^{\mathrm{c}}$, Linsheng Song ${ }^{\mathrm{b}, *}$
}

\begin{abstract}
A C-type lectin of multiple CRDs (CfLec-4) from Chlamys farreri was selected to investigate the sequence variation and functional differentiation of its CRDs. Its four CRDs with EPD/LSD, EPN/FAD, EPN/LND and EPN/YND key motifs were recombined separately. The recombinant proteins of CRD1 and CRD2 (designated as rCRD1 and rCRD2) could bind LPS and mannan, while the recombinant proteins of CRD3 and CRD4 (designated as rCRD3 and rCRD4) could bind LPS, PGN, mannan and glucan. Moreover, rCRD3 displayed broad microbe binding spectrum towards Gram-positive bacteria Staphylococcus aureus and Micrococcus luteus, Gram-negative bacteria Escherichia coli and Vibrio anguillarum, as well as fungi Pichia pastoris and Yarrowia lipolytica. These results indicated CRD3 contributed more to CfLec-4's nonself-recognition ability. Furthermore, CRD1, CRD3 and CRD4 functioned as opsonin participating in the clearance against invaders in scallops. The sequence variation in $\mathrm{Ca}^{2+}$ binding site 2 among CRDs was suspect to be associated
\end{abstract}


with such functional differentiation.

Keywords: Chlamys farreri; C-type lectin; carbohydrate-recognition domain; $\mathrm{Ca}^{2+}$ binding site 2; functional differentiation

\section{Introduction}

C-type lectins are a superfamily of $\mathrm{Ca}^{2+}$-dependent carbohydrate-recognition proteins. They function as PRR to discriminate self and nonself by recognizing and binding the terminal sugars from various microbes (Weis et al., 1998; Zelensky and Gready, 2005). The carbohydrate recognition domains (CRDs) endow C-type lectins with activities of nonself-recognition and clearance of invaders. Recently, C-type lectins have been assorted into 17 subgroups in vertebrates according to their structural and functional similarities (Zelensky and Gready, 2005), in which collectin is one subgroup serving as PRR to discriminate self and nonself (Cambi and Figdor, 2003; Medzhitov and Janeway, 2002), while selectin mainly mediates cellular adhesion to activate encapsulation and inflammation (Patel et al., 2002). For instance, mannose-binding lectin (MBL) belonging to collectin subgroup can recognize and bind terminal mannose residues and initiate the lectin pathway of complement system (Endo et al., 2006; Fujita, 2002). And P-selectin in selectin subgroup can attract and enrich leukocytes to the site of infection to quickly eliminate the pathogenic bacteria.

There are 1-4 $\mathrm{Ca}^{2+}$ binding sites in each CRD, among which $\mathrm{Ca}^{2+}$ binding site 2 has been proved to determine the binding specificity of C-type lectins in vertebrates (Weis et al., 1991; Weis and Drickamer, 1996; Weis et al., 1998; Zelensky and Gready, 
2005). Briefly, there are two motifs existing in $\mathrm{Ca}^{2+}$ binding site 2 . The first motif is EPN (Glu-Pro-Asn) or QPD (Gln-Pro-Asp), which is experimentally verified to determine the binding specificity of C-type lectin. The second motif is WND (Trp-Asn-Asp) which is identified to cooperate with the first motif in binding process against carbohydrates (Cambi et al., 2005; Weis and Drickamer, 1996; Weis et al., 1998). Moreover, intensive researches demonstrated that C-type lectins with EPN/WND motifs bind D-mannose or similar sugar, while C-type lectins with QPD/WND motifs bind D-galactose or similar sugar (Wang et al., 2011a; Zelensky and Gready, 2005). However, the two motifs are of great diversity of characteristics in invertebrate, and various kinds of amino acid sequence have been found in the corresponding sites of two specific motifs. For instance, EPD (Glu-Pro-Asp), QPG (Gln-Pro-Gly), QPS (Gln-Pro-Ser), YPG (Tyr-Pro-Gly), and YPT (Tyr-Pro-Thr) were found in the first motif, at the same time WID (Trp-Ile-Asp), WSD (Trp-Ser-Asp), WHD (Trp-His-Asp), FSD (Phe-Ser-Asp) and LSD (Leu-Ser-Asp) were found in the second motif (Wang et al., 2011a).

The number and organization of CRDs determine the affinity and spectrum of lectins to recognize and bind nonself invaders. Some vertebrate C-type lectins contained several CRDs and single CRD containing C-type lectins usually tend to form polymers to perform functions. For instance, the mannose receptor is composed of eight CRDs, whereas MBL is inclined to form polymers to activate complement system (Zelensky and Gready, 2005). To our knowledge, most reported invertebrate C-type lectins containe one single CRD (Ao et al., 2007; Belogortseva et al., 1998; 
Huang et al., 2015; Li et al., 2015; Mu et al., 2012). Very recently, however, several invertebrate C-type lectins with double or multiple CRDs have been proved to involve in immune responses including PAMPs and microbes binding (Yang et al., 2015), bacteria agglutination (Tian et al., 2009) and opsonization (Wang et al., 2011b). Fc-Lec2 from Fenneropenaeus chinensis with two tandem CRDs and CfLec-3 from $C$. farreri with three dissimilar CRDs have broad and strong affinity to recognize microbes and PAMPs (Yang et al., 2015; Zhang et al., 2009). Nevertheless, the relationship between the structure and functions of multidomain C-type lectins is still defectively understood, especially in invertebrates.

In our previous study, CfLec-4 with four CRDs was demonstrated to be involved in PAMPs recognition, microbe binding and phagocytosis enhancement against bacteria. In the current study, the four CRDs of CfLec-4 were expressed in Escherichia coli separately, and their PAMP binding specificities, microbe binding spectrums as well as the phagocytosis enhancement activities were examined to comprehensively explore the role of each CRD played in immune response against invaders.

\section{Materials and methods}

\subsection{Scallop and microbes}

Adults of scallop C. farreri with an average $55 \mathrm{~mm}$ of shell length were collected from a farm in Qingdao, Shandong Province, China, and maintained in the aerated seawater at $18^{\circ} \mathrm{C}$ for a week before processing. 
purchased from Microbial Culture Collection Center (Beijing, China). Vibrio anguillarum was kindly provided by Dr. Zhaolan Mo. Yarrowia lipolytica was kindly provided by Dr. Zhenming Chi. Pichia pastoris GS115 was purchased from Invitrogen.

\subsection{Sequence analysis}

The ClustalW Multiple Alignment program (http://www.ebi.ac.uk/clustalw/) was used to create the multiple sequence alignment. An unrooted phylogenetic tree was constructed based on the sequence alignment by the neighbor-joining (NJ) algorithm using the Mega4.0 program (Wang et al., 2015). Bootstrap re-sampling (1000 pseudo-replicates) was employed to test the reliability of the branching.

\subsection{Preparation of recombinant proteins}

The four CRDs of CfLec-4 were recombined to plasmid pET-32a and were transformed into the strains $E$. coli BL21(DE3)-pLysS respectively. The recombinant protein rCRD1, rCRD2, rCRD3, rCRD4 and the recombinant protein Trx (designated as rTrx) were purified and quantified as described before (Huang et al., 2012).

\subsection{Preparation of antibodies and Western-blot analysis}

The renatured protein rCfLec- 4 and rTrx were immuned to 6 weeks old mice to acquire polyclonal antibody. The specificity of antibodies were detected as described previously (Huang et al., 2012). 

and $V$. anguillarum) and fungi ( $P$. pastoris and $Y$. lipolytica) were used to determine the microbe binding spectrum of $\mathrm{rCRD} 1, \mathrm{rCRD} 2, \mathrm{rCRD} 3$ and $\mathrm{rCRD} 4$ according to the method described by Lee (Lee and Söderhäll, 2001). One hundred microliters of rCRD1, rCRD2, rCRD3 and rCRD4 were incubated with microbes under rotations for $30 \mathrm{~min}$ at room temperature. Then the microbes were washed with TBS and finally, subjected to elution with $8 \%$ SDS. The microbe binding assay was detected by Western-blot analysis. rTrx group was employed as negative control.

\subsection{The PAMPs binding assay}

The PAMP binding activities of rCRD1, rCRD2, rCRD3, and rCRD4 were measured according to previous report (Huang et al., 2012). After incubated with primary antibody and second antibody, one hundred microliters of $0.1 \%(\mathrm{w} / \mathrm{v})$ pnitrophenyl phosphate (pNPP) containing $0.5 \mathrm{mM} \mathrm{MgCl}_{2}$ was added to each well for chromogenic reaction. The same concentration of rTrx was used as control. The wells filled with $100 \mu \mathrm{L}$ of TB were used as blank. The absorbance was measured with an automatic ELISA reader at $405 \mathrm{~nm}$. Each experiment was repeated in triplicates and the results were given in terms of the mean of three individual measurements \pm S.E. $(N=3)$. Samples with $\mathrm{P}$ (sample)-B (blank)/N (negative)-B (blank) $>2.1$ were considered as positive.

\subsection{Phagocytosis assay}

Phagocytosis assay was performed according to previous method (Huang et al., 2012). The phagocytic activity of hemocyte attached to the slide was measured using a light microscope. Two hundred hemocytes on each slide were counted, and the 
phagocytic rate (PR) representing the phagocytic activities was expressed as following: $\mathrm{PR}=($ phagocytic hemocytes $) /($ total hemocytes $) \times 100 \%$. For each treatment, assay was performed in three different slides for statistical analysis.

\section{Results}

\subsection{The sequence features and phylogeny of CRD1, CRD2, CRD3 and CRD4}

Multiple sequence alignment of CRDs was developed by ClustalW to identify the signature sequences of the four CRDs (Fig. 1). Each CRD contained four cysteine residues involving in the formation of the conserved internal disulfide bridges. Besides, the two N-terminus cysteine residues in all CRDs indicated that they were of long-form (Zelensky and Gready, 2005). The key motifs of $\mathrm{Ca}^{2+}$ binding site 2 in four CRDs were EPD/LSD, EPN/FAD, EPN/LND and EPN/YND, respectively. A phylogenetic tree was constructed by NJ method with 1000 bootstrap-test based on the multiple alignments of CRDs from bivalve lectins and other C-type lectins from Botryllus schlosseri, Danio rerio, Gallus gallus, Homo sapiens, Mus musculus, Rattus norvegicus, Salmo salar and Taeniopygia guttata (Fig. 2). Three distinct groups were separated in the phylogenetic tree. The CRDs of collectins were clustered into a branch and the CRDs of selectins formed as another branch. The four CRDs of CfLec-4, CfLec-1 and AiCTL-7 from scallops clustered into a branch.

\subsection{The recombinant proteins and antibodies of CRD1, CRD2, CRD3 and CRD4}

The whole cell lysate of E. coli BL21 (DE3)-pLysS with pET-32a-CRD1, -CRD2, 
-CRD3 and -CRD4 induced by IPTG were analyzed by SDS-PAGE. A distinct band with molecular weight of $25 \mathrm{kDa}$ was revealed and was consistent with the predicted molecular mass (Fig. 3A, lane 3, 7, 11 and 15). Four clear bands were revealed in the Western-blot analysis with anti-CfLec-4 polyclonal antibody (Fig. 3A, lane 4, 8, 12 and 16). In the control of E. coli BL21 (DE3)-pLysS with pET-32a, there was clear band corresponding to rTrx with $18 \mathrm{kDa}$ molecular weight (Fig. 3B).

\subsection{PAMPs binding activities of $r C R D 1, r C R D 2, r C R D 3$ and $r C R D 4$}

All the rCRDs of CfLec-4 exhibited a dose-dependent binding capability towards the different tested PAMPs. rCRD1 and rCRD2 bound LPS and mannan while rCRD3 and rCRD4 bound four kinds of PAMPs including LPS, PGN, mannan and glucan. Furthermore, the binding affinity of four rCRDs was different from each other. rCRD3 and rCRD4 exhibited the stronger binding affinity for LPS, PGN and glucan, indicating that rCRD3 and rCRD4 were the major recognition elements of CfLec-4. As negative control, rTrx could not bind any tested PAMPs (data not shown).

\subsection{Microbe binding spectrum of $r C R D 1, r C R D 2, r C R D 3$ and $r C R D 4$}

In order to furthur confirm the interaction between CRDs and microorganism, a direct binding assay was carried out to determine the binding activity of the four rCRDs against Gram-negative bacteria, Gram-positive bacteria and fungi. The binding spectrums of rCRD1 and rCRD2 were the same and they could bind $V$. auguillarum and S. aureus. rCRD3 exhibited the widest microbe binding spectrum and the clear bands were detected in all microbes including E. coli, V. auguillarum, $S$. 
aureus, M.luteus, P. pastoris and Y. lipolytica (Fig. 5). As for rCRD4, there was only a clear band detected in S. aureus (Fig. 5).

\subsection{The phagocytosis enhancement of $r C R D 1, r C R D 2, r C R D 3$ and $r C R D 4$}

The phagocytosis enhancement assay of rCRD1, rCRD2, rCRD3 and rCRD4 was performed with scallop hemocytes. The phagocytic rate of rCRD1, rCRD2, rCRD3 and rCRD4 groups was $28.7 \%, 22.1 \%, 32.7 \%$ and $25.3 \%$, repectively. While the phagocytic rate of TBS and rTrx group was $17.0 \%$ and $18.1 \%$, respectively (Fig. 6). Compared with TBS and rTrx groups, rCRD1, rCRD3 and rCRD4 could significantly enhance $(P<0.01)$ the phagocytic activity of scallop hemocytes against $E$. coli.

\section{Discussion}

C-type lectins play key roles in defense against pathogen infection by serving as PRR in innate immunity (Akira et al., 2006; Cambi and Figdor, 2003; Weis et al., 1998). The immune functions of C-type lectin mainly rely on its characteristic CRDs, which can bind various carbohydrates through $\mathrm{Ca}^{2+}$ binding site 2 . Some multidomain C-type lectins have been reported to involve in pathogen recognition (Akira et al., 2006; Cambi et al., 2005; Geijtenbeek et al., 2004; Vasta et al., 2007; Zelensky and Gready, 2005), cellular adhesion (Kerrigan and Brown, 2009), antigen uptake (Kang et al., 2004) and complement pathway activation in vertebrates (Endo et al., 2006; Fujita, 2002). Recently, C-type lectins with multidomain have also been identified and characterized in invertebrates, such as BmMBP (Watanabe et al., 2006), CfLec-3 
(Yang et al., 2015) and Fc-Lec2 (Zhang et al., 2009). But their immune functions and the detailed mechanism were still not well understood. In the present study, the four CRDs of CfLec-4 were recombined separately, and their immune functions were thoroughly studied to comprehensively understand the immune response mechanism of multidomain C-type lectin in invertebrate.

So far, many C-type lectins identified from invertebrates exhibited nonself recognizing and binding activity against certain PAMPs and microbes (Hatakeyama et al., 2011; Wang et al., 2011a). For instance, AmphiCTL1 from Branchiostoma belcheri could bind PGN and glucan (Yu et al., 2007), and BmMBP could interact with LTA, PGN and mannan (Watanabe et al., 2006). Interestingly, the C-type lectins with multidomain were found to possess extensive nonself-recognition and binding spectrum. For example, CfLec-3 with three CRDs from C. farreri possessed wide PAMPs and microbes binding spectrum (Yang et al., 2015), and Fc-Lec2 with two CRDs from Litopeneaus vannamei displayed strong binding affinity against microbes and polysaccharide (Zhang et al., 2009). However, the wide nonself-reconition machanism of multidomain C-type lectins were still not well documented. In the current study, the binding ability and binding spectrum towards PAMPs and microbe of the four CRDs in CfLec- 4 were examined to better understand the possible binding mechanism. rCRD3 and rCRD4 could bind all the tested PAMPs, while rCRD1 and rCRD2 only bound LPS and mannan. Moreover, rCRD3 and rCRD4 exhibited stronger binding affinity to LPS, PGN and glucan compared with other rCRDs. It could be inferred that CRD3 and CRD4 were the dominant recognition CRDs of 
CfLec-4 and there was function differentiation among the four CRDs of CfLec-4. Meanwhile, rCRD3 had the widest microbe binding spectrum among the four rCRDs of CfLec-4. It was consistent with its PAMPs binding spectrum. rCRD1and rCRD2 had the same microbe binding spectrum to V. auguillarum and S. aureus while rCRD4 could only bind to S. aureus, which was not corresponding with their PAMPs binding spectrum. This phenomenon was also found in AiCTL-3 from A. irradians (Huang et al., 2013). AiCTL-3 could bind to PGN, so it was inferred to bind S. aureus and M. luteus. However, it could only bind to S. aureus other than M. luteus. The mechanism behind this phenomenon needs further research.

It is accepted that C-type lectins act not only as PRR to bind PAMPs but also as opsonin to promote the phagocytosis of hemocytes against bacteria (Yang et al., 2010; Yang et al., 2011). Recently, several vertebrate C-type lectins, such as DC-SIGN, Dectin-1 and mannose receptor, were found to trigger the opsonization after interacting with microbes (Geijtenbeek et al., 2000; Yang et al., 2015), and some invertebrate C-type lectins were also found to initiate opsonization against bacteria and parasites (Wang et al., 2011a; Wang et al., 2011b). In our previous report, CfLec-4 was found to enhance the phagocytosis ability of hemocytes after binding PAMPs and adhering to the cell surface of certain bacteria (Huang et al., 2012). In the present study, rCRD1, rCRD3 and rCRD4 exhibited the activity to trigger the phagocytosis of hemocytes, indicating that not all the CRDs of CfLec-4 were necessary in the process of triggering opsonization against invaders. CRD1, CRD3 and CRD4 were the main contributor of the opsonization triggered by CfLec- 4 . 

the binding specificity of CRD, which is composed of two conserved characteristic motifs. The first one is EPN or QPD, which is the carbohydrate binding site and also the key motif determining the carbohydrate binding specificity. For example, C-type lectin with EPN motif could bind D-mannose or similar sugar, whereas those with QPD motif bind D-galactose or similar sugar in vertebrates. The second conserved motif in $\mathrm{Ca}^{2+}$ binding site 2 is WND which has been proved to assist the binding between CRD and certain carbohydrates. The position of hydrogen bond donors and acceptors in the two motifs of $\mathrm{Ca}^{2+}$-binding site 2 determined C-type lectins' binding

259 specificity. However, accumulating evidences have demonstrated that the $\mathrm{Ca}^{2+}$ binding site 2 in invertebrate C-type lectins are of diverse structure and multifunction.

The first key motif in $\mathrm{Ca}^{2+}$ binding site 2 seems to be more various in invertebrates. For example, there were EPN, EPD, QPG, QPS, YPT and YPG in bivalve C-type lectins, which were supposed to bind diversified carbohydrates (Wang et al., 2011a).

PAMPs binding spectrums were examined in order to find the possible PAMPs 
CRD3 and CRD4 with EPN motif displayed different PAMPs binding ability. In detail, rCRD2 could bind LPS and mannan while rCRD3 and rCRD4 could bind all PAMPs tested. It was a new discovery in bivalve C-type lectin which was not corresponding with the binding rules in that of vertebrates. It was noticeable that the second key motif of $\mathrm{Ca}^{2+}$ binding site 2 in CRD2, CRD3 and CRD4, which were FAD, LND and YND respectively, which were quite different from the WND motif in vertebrates. The asparagine and aspartate were involved in hydrogen bond formation with carbohydrates. Thus, the replacement of amino acids in two key motifs might change the hydrogen bond formation involved in carbohydrates binding, and thus affect the binding specificity of $\mathrm{Ca}^{2+}$ binding site 2 which worth further investigation.

\section{Acknowledgements}

The authors would thank all of the colleagues in our lab for helpful discussion and technical advice. This research was supported by National High Technology Research and Development Program (863 Program, No. 2014AA093501) from the Chinese Ministry of Science and Technology, grants (No. 31530069 to L.S., No. 41006096 to H.Z.) from National Science Foundation of China, and Dalian high level talent innovation support program (No. 2015R020). Thanks for Dr. Zhenming Chi for kindly providing Yarrowia lipolytica.

\section{References}

Akira, S., Uematsu, S., Takeuchi, O., 2006. Pathogen recognition and innate immunity. Cell 124, 783-801.

Ao, J., Ling, E., Yu, X., 2007. Drosophila C-type lectins enhance cellular encapsulation. Mol. 
Immunol. 44, 2541-2548.

Belogortseva, N.I., Molchanova, V.I., Kurika, A.V., Skobun, A.S., Glazkova, V.E., 1998. Isolation and characterization of new GalNAc/Gal-specific lectin from the sea mussel Crenomytilus grayanus. Comp. Biochem. Physiol., C: Toxicol. Pharmacol.119C, 45-50.

Cambi, A., Figdor, C.G., 2003. Dual function of C-type lectin-like receptors in the immune system. Curr. Opin. Cell Biol. 15, 539-546.

Cambi, A., Koopman, M., Figdor, C.G., 2005. How C-type lectins detect pathogens. Cell. Microbiol. 7, 481-488.

Endo, Y., Takahashi, M., Fujita, T., 2006. Lectin complement system and pattern recognition. Immunobiology 211, 283-293.

Fujita, T., 2002. Evolution of the lectin-complement pathway and its role in innate immunity. Nat. Rev. Immunol. 2, 346-353.

Geijtenbeek, T.B., Kwon, D.S., Torensma, R., van Vliet, S.J., van Duijnhoven, G.C., Middel, J., Cornelissen, I.L., Nottet, H.S., KewalRamani, V.N., Littman, D.R., 2000. DC-SIGN, a dendritic cell-specific HIV-1-binding protein that enhances trans-infection of T cells. cell 100, 587-597.

Geijtenbeek, T.B.H., van Vliet, S.J., Engering, A., 't Hart, B.a., van Kooyk, Y., 2004. Self- and nonself-recognition by C-type lectins on dendritic cells. Annu. Rev. Immunol. 22, 33-54.

Hatakeyama, T., Kamiya, T., Kusunoki, M., Nakamura-Tsuruta, S., Hirabayashi, J., Goda, S., Unno, H., 2011. Galactose recognition by a tetrameric C-type lectin, CEL-IV, containing the EPN carbohydrate recognition motif. J. Biol. Chem. 286, 10305-10315.

Huang, M., Song, X., Zhao, J., Mu, C., Wang, L., Zhang, H., Zhou, Z., Liu, X., Song, L., 2013. A C-type lectin (AiCTL-3) from bay scallop Argopecten irradians with mannose/galactose binding ability to bind various bacteria. Gene 531, 31-38.

Huang, M., Wang, L., Yang, J., Zhang, H., Wang, L., Song, L., 2012. A four-CRD C-type lectin from Chlamys farreri mediating nonself-recognition with broader spectrum and opsonization. Dev. Comp. Immunol. 39, 363-369.

Huang, M., Zhang, H., Jiang, S., Wang, L., Liu, R., Yi, Q., Song, L., 2015. An EPD/WSD motifs containing C-type lectin from Argopectens irradians recognizes and binds microbes with broad spectrum. Fish Shellfish Immunol. 43, 287-293. 
Kang, Y.-S., Kim, J.Y., Bruening, S.a., Pack, M., Charalambous, A., Pritsker, A., Moran, T.M., Loeffler, J.M., Steinman, R.M., Park, C.G., 2004. The C-type lectin SIGN-R1 mediates uptake of the capsular polysaccharide of Streptococcus pneumoniae in the marginal zone of mouse spleen. Proc. Natl. Acad. Sci. USA. 101, 215-220.

Kerrigan, A.M., Brown, G.D., 2009. C-type lectins and phagocytosis. Immunobiology 214, $562-575$.

Lee, S.Y., Söderhäll, K., 2001. Characterization of a pattern recognition protein, a masquerade-like protein, in the freshwater crayfish Pacifastacus leniusculus. J. Immunol. 166, 7319-7326.

Li, H., Zhang, H., Jiang, S., Wang, W., Xin, L., Wang, H., Wang, L., Song, L., 2015. A single-CRD C-type lectin from oyster Crassostrea gigas mediates immune recognition and pathogen elimination with a potential role in the activation of complement system. Fish Shellfish Immunol. 44, 566-575.

Medzhitov, R., Janeway, C.a., 2002. Decoding the patterns of self and nonself by the innate immune system. Science 296, 298-300.

Mu, C., Song, X., Zhao, J., Wang, L., Qiu, L., Zhang, H., Zhou, Z., Wang, M., Song, L., Wang, C., 2012. A scallop C-type lectin from Argopecten irradians (AiCTL5) with activities of lipopolysaccharide binding and Gram-negative bacteria agglutination. Fish Shellfish Immunol. $32,716-723$.

Patel, K.D., Cuvelier, S.L., Wiehler, S., 2002. Selectins: critical mediators of leukocyte recruitment. Semin. Immunol. 14, 73-81.

Tian, Y.Y., Liu, Y., Zhao, X.F., Wang, J.X., 2009. Characterization of a C-type lectin from the cotton bollworm, Helicoverpa armigera. Dev. Comp. Immunol. 33, 772-779.

Vasta, G., Ahmed, H., Tasumi, S., Odom, E., Saito, K., 2007. Biological roles of lectins in innate immunity: molecular and structural basis for diversity in self/non-self recognition. Current topics in innate immunity, 389-406.

Wang, L., Huang, M., Zhang, H., Song, L., 2011a. The immune role of C-type lectins in molluscs. ISJ 8, 241-246.

Wang, L., Wang, L., Zhang, D., Jiang, Q., Sun, R., Wang, H., Zhang, H., Song, L., 2015. A novel multi-domain C1qDC protein from Zhikong scallop Chlamys farreri provides new insights into the function of invertebrate C1qDC proteins. Dev. Comp. Immunol. 52, 202-214. 
Wang, L., Yang, J., Zhang, H., Huang, M., Kong, P., Zhou, Z., Song, L., 2011b. A multi-CRD C-type lectin with broad recognition spectrum and cellular adhesion from Argopecten irradians. Dev. Comp. Immunol. 36 (3), 591-601.

Watanabe, A., Miyazawa, S., Kitami, M., Tabunoki, H., Ueda, K., Sato, R., 2006. Characterization of a novel C-type lectin, Bombyx mori multibinding protein, from the B. mori hemolymph: mechanism of wide-range microorganism recognition and role in immunity. J. Immunol. 177, 4594-4604.

Weis, W., Kahn, R., Fourme, R., Drickamer, K., Hendrickson, W., 1991. Structure of the calcium-dependent lectin domain from a rat mannose-binding protein determined by MAD phasing. Science 254, 1608-1615.

Weis, W.I., Drickamer, K., 1996. Structural basis of lectin-carbohydrate recognition. Annu. Rev. Biochem. 65, 441-473.

Weis, W.I., Taylor, M.E., Drickamer, K., 1998. The C-type lectin superfamily in the immune system. Immunol. Rev. 163, 19-34.

Yang, J., Huang, M., Zhang, H., Wang, L., Wang, H., Wang, L., Qiu, L., Song, L., 2015. CfLec-3 from scallop: an entrance to non-self recognition mechanism of invertebrate C-type lectin. Sci. Rep. 5, e10068.

Yang, J., Qiu, L., Wei, X., Wang, L., Zhou, Z., Zhang, H., Liu, L., Song, L., 2010. An ancient C-type lectin in Chlamys farreri (CfLec-2) that mediate pathogen recognition and cellular adhesion. Dev. Comp. Immunol. 34, 1274-1282.

Yang, J., Wang, L., Zhang, H., Qiu, L., Wang, H., Song, L., 2011. C-type lectin in Chlamys farreri (CfLec-1) mediating immune recognition and opsonization. PLoS One 6, e17089.

Yu, Y., Huang, H., Feng, K., Pan, M., Yuan, S., Huang, S., Wu, T., Guo, L., Dong, M., 2007. A short-form C-type lectin from amphioxus acts as a direct microbial killing protein via interaction with peptidoglycan and glucan. J. Immunol. 179, 8425-8434.

Zelensky, A.N., Gready, J.E., 2005. The C-type lectin-like domain superfamily. FEBS J. 272, 6179-6217.

Zhang, X.W., Xu, W.T., Wang, X.W., Mu, Y., Zhao, X.F., Yu, X.Q., Wang, J.X., 2009. A novel C-type lectin with two CRD domains from Chinese shrimp Fenneropenaeus chinensis functions as a pattern recognition protein. Mol. Immunol. 46, 1626-1637. 


\section{Figure legends}

Fig. 1

Multiple sequence alignment by ClustalW of four CRDs from CfLec-4 with other genes' CRDs. These gene include CfLec-1 (GenBank accession no. DQ209290) and CfLec-2 (DQ209289) of C. farreri, AiCTL-7 from A. irradians. Amino acid residues that are conserved in at least $50 \%$ sequences are shaded in dark, and similar amino acids are shaded in grey. Conserved cysteine residues involved in the formation of the CRD internal disulfide bridges were marked with black square, whereas two extra cysteine residues in long-form CRD were marked with black triangle. The letters in box were $\mathrm{Ca}^{2+}$ binding site 2 of CRDs which determined the ligand binding specificity.

\section{Fig. 2}

Unrooted phylogenetic tree of the CRDs in CfLec-4 (CRD1, CRD2, CRD3 and CRD4) with those in other C-type lectins. The tree is constructed by the neighbor-joining (NJ) algorithm using the Mega4.0 program based on the multiple sequence alignment by ClustalW. Bootstrap values of 1000 replicates (\%) are indicated for the branches. The scale bar corresponds to 0.2 estimated amino-acid substitutions per site. The CRDs sequences used for phylogenetic analysis are as follows, 1: NP_002996 (H. sapiens P-selectin); 2: AAI09160 (P-selectin M. musculus); 3: NP_035476 (L-selectin $M$. musculus); 4: CAI19356.1 (L-selectin H. sapiens); 5: CAX13223.1 (L-selectin D. rerio); 6: XP_002190413 (L-selectin T. guttata); 7: AAI31552 (E-selectin H. sapiens); 8: AAA37577 (E-selectin M. musculus); 9: CAA62217 (MBP B. schlosseri); 10: 
sapiens); 13: NP_075623 (SP-A M. musculus); 14: NP_989937 (SP-A G. gallus); 15: CAA34079 (MBP-A H. sapiens); 16: P19999 (MBP-A $R$. norvegicus); 17: NP_033186 (SP-D M. musculus); 18: AAB59450 (SP-D H. sapiens); 19: HM149769 (AiCTL-7 A. irradians); 20: DQ209290 (CfLec-1 C. farreri); 21: CRD1 of CfLec-4; 22: CRD2 of CfLec-4; 23: CRD3 of CfLec-4; 24: CRD4 of CfLec-4.

Fig. 3

SDS-PAGE and Western-blot analysis of rCRD1, rCRD2, rCRD3, rCRD4 and rTrx. (A) Lane M: protein molecular standard; lane 1: negative control for rCRD1 (without induction); lane 2: induced rCRD1; lane 3: purified rCRD1; lane 4: Western-blot based on the sample of lane 3; lane 5: negative control for rCRD2; lane 6: induced rCRD2; lane 7: purified rCRD2; lane 8: Western-blot based on the sample of lane 7; lane 9: negative control for rCRD3; lane 10: induced rCRD3; lane 11: purified rCRD3; lane 12: Western-blot based on the sample of lane 11; lane 13: negative control of rCRD4; lane 14: induced rCRD4; lane 15: purified rCRD4; lane 16: Western-blot based on the samples of lane 15. (B) Lane M: protein molecular standard; lane 1: induced rTrx; lane 2: negative control for rTrx (without induction); lane 3: purified rTrx; lane 4: Western-blot based on the sample of lane 3.

\section{Fig. 4}

ELISA analysis of the interaction between four rCRDs and the PAMPs. Plates were coated with four kinds of PAMPs including glucan, LPS, PGN and mannan. Then incubated with different amounts of rCRDs and $\mathrm{rTrx}$ at $18^{\circ} \mathrm{C}$ for $3 \mathrm{~h}$. After incubated with rat polyclonal antiserum, the interaction was detected with goat-anti-rat Ig-alkaline phosphatase conjugate at $405 \mathrm{~nm}$. Samples with $\mathrm{P} / \mathrm{N}>2.1$ were considered 
positive. Results are representative of the mean of three replicates \pm SE. The significant difference between CRDs were marked with letters, and different letters were used for significant difference $(P<0.05)$ between CRDs.

\section{Fig. 5}

The microbe binding spectrum of rCRD1, rCRD2, rCRD3 and rCRD4 revealed by Western-blot. One hundred microlitres of purified rCRD1, rCRD2, rCRD3 and rCRD4 in TBS $\left(100 \mu \mathrm{g} \mathrm{mL}^{-1}\right)$ was incubated with microorganisms under rotation for $30 \mathrm{~min}$ at room temperature. Microorganisms were pelleted, washed three times with TBS, resuspended in $50 \mu \mathrm{L}$ of TBS, and then subjected to elution with $8 \%$ SDS. The supernatant was collected. The binding spectrum of rCRD1, rCRD2, rCRD3 and rCRD4 was detected by Western-blot.

Fig. 6

Phagocytosis enhanced by rCRD1, rCRD2, rCRD3 and rCRD4. The hemocytes, rCRDs and rTrx were resuspended with TBS buffer, respectively. The hemocytes were incubated with TBS, rTrx and rCRDs for $30 \mathrm{~min}$, then E. coli was added into each suspension for another $1 \mathrm{~h}$. The mixture was mounted onto a glass slide and stained with Giemsa. The phagocytic activity of hemocyte was measured using a light microscope. Two hundred hemocytes on each slide were counted. Phagocytic rate (PR) $=($ phagocytic hemocytes $) /($ total hemocytes $) \times 100 \%$. For each treatment, assay was performed in three different slides for statistic analysis. The columns represent the mean of three individual counts \pm S.E.M, student's $t$ test, error-bars represent biological repeats $(* *: P<0.01)$. 
CRD1

CRD2

CRD3

CRD4

CfLec-2

AiCTL-7

Cflec-1

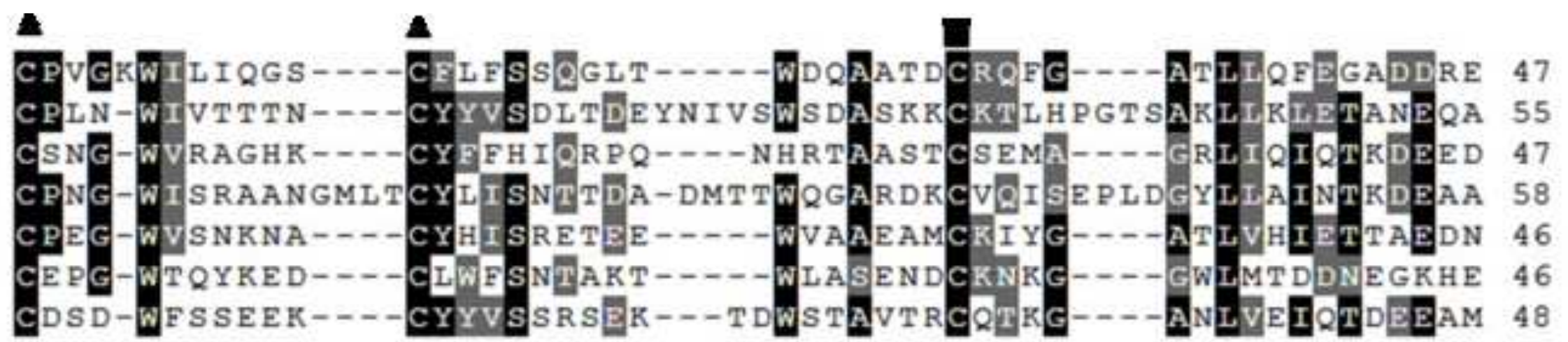

CRD1 LFTRTIVN----MTSERWWTDITDYNHPGGWSWGEDDSEITANPGAVVWN-VEPDPRHH 102

CRD2 YIRAQLAE--LQMTDQLYWIGMSDQAHEGHWTWDD--GSPVNQS-NIEWT-AEPNNLGGT

CRD3 WLRVQTLR----YDSYAFWTGLI FQPSSSSWVWNT-- -DTKANMSIINWN-QEPNN-EGN

CRD4 FIAHALKN--ISQIATGWWTGLNDKKVEGYWEYDTAFNNPVQNN-VIPWD-GEPNNIGGT 109

FLeC-2 FISEYLRNNSIVYNDHQYWIGLSDWEFEGTFIWVP---EGVTPG-YTNWGPGEPDNNHQN AiCTL-7 EISTIMYA-FRNFHFNKEFIGGSDTAFENVWRWLE---TGINVGPFSKWGTGEPDGNTTK CfLec-1 IIMRNLPS--RVSRADIIYTG-RKRNDNRDWVELTN--EKIVDTSVRSWGSGEPDGGSQN 98 114 102 102 103

CRD1

CRD2 EN 135 SQC-AIIY YN- - GR - EADADC- -

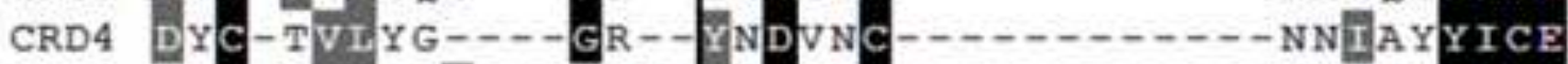
124 139

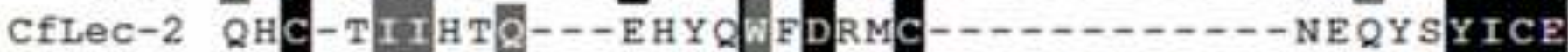
130 AiCTL-7 N-CLALRWEND--RDLVWSDESCGHVSTSHнHнHGHGLLNY ICE 143

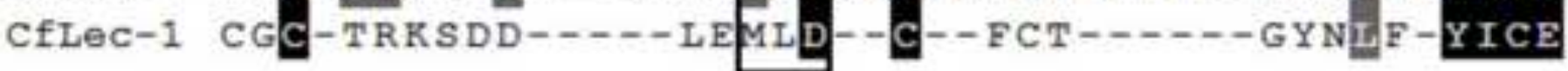




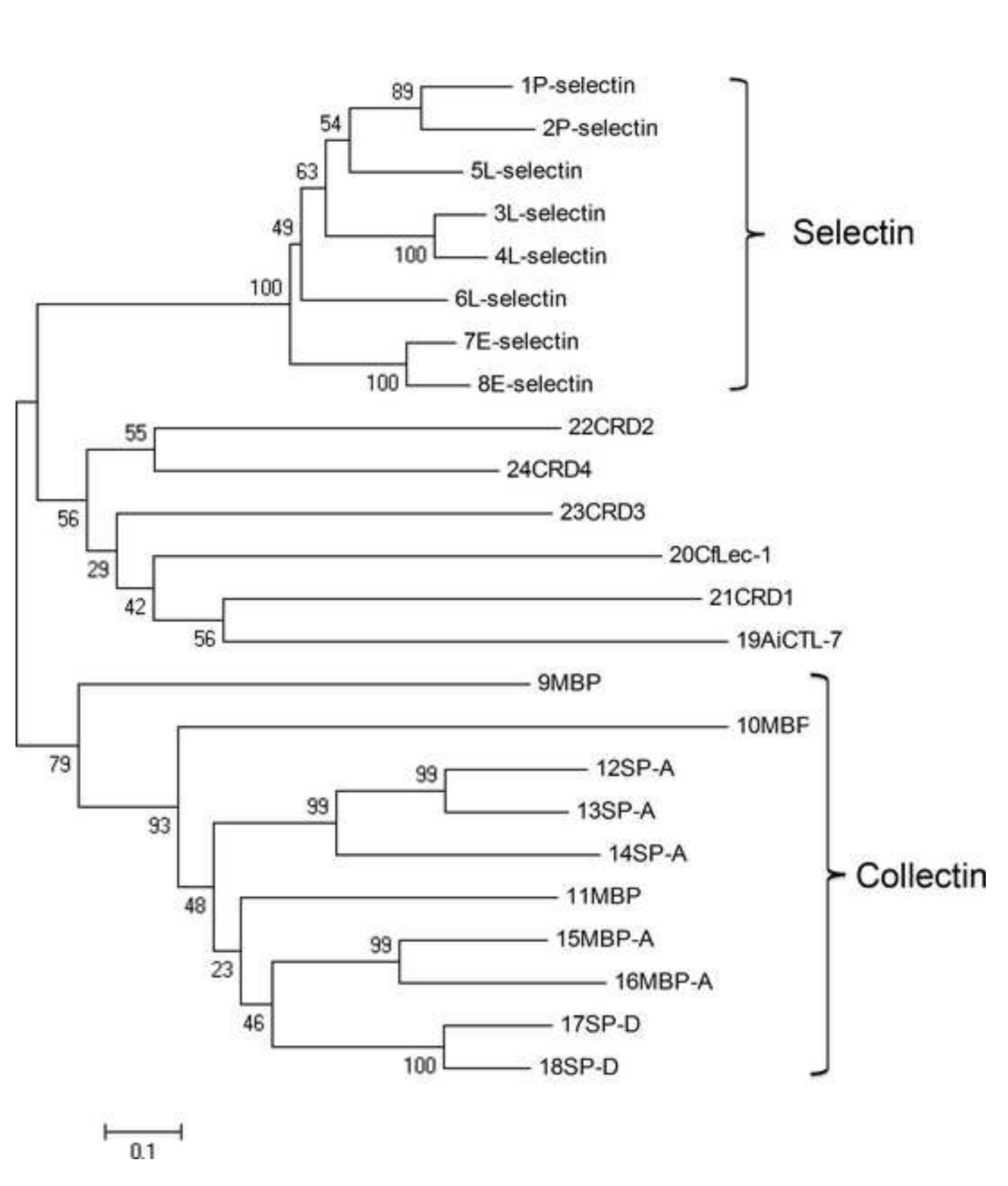

Figure 2

.

thater

Figure

.

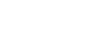

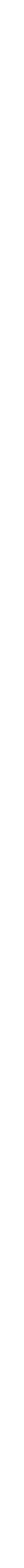

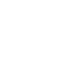

列

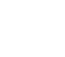

.

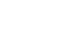

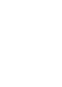




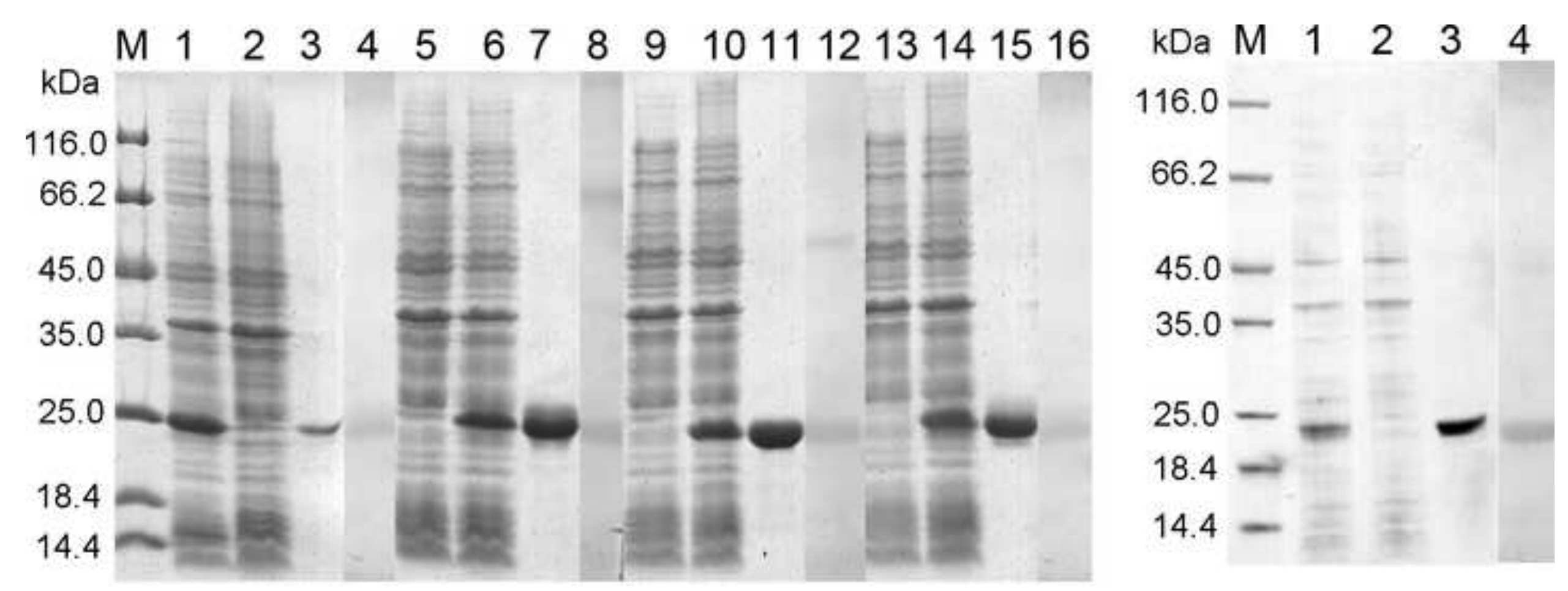




\section{Glucan}
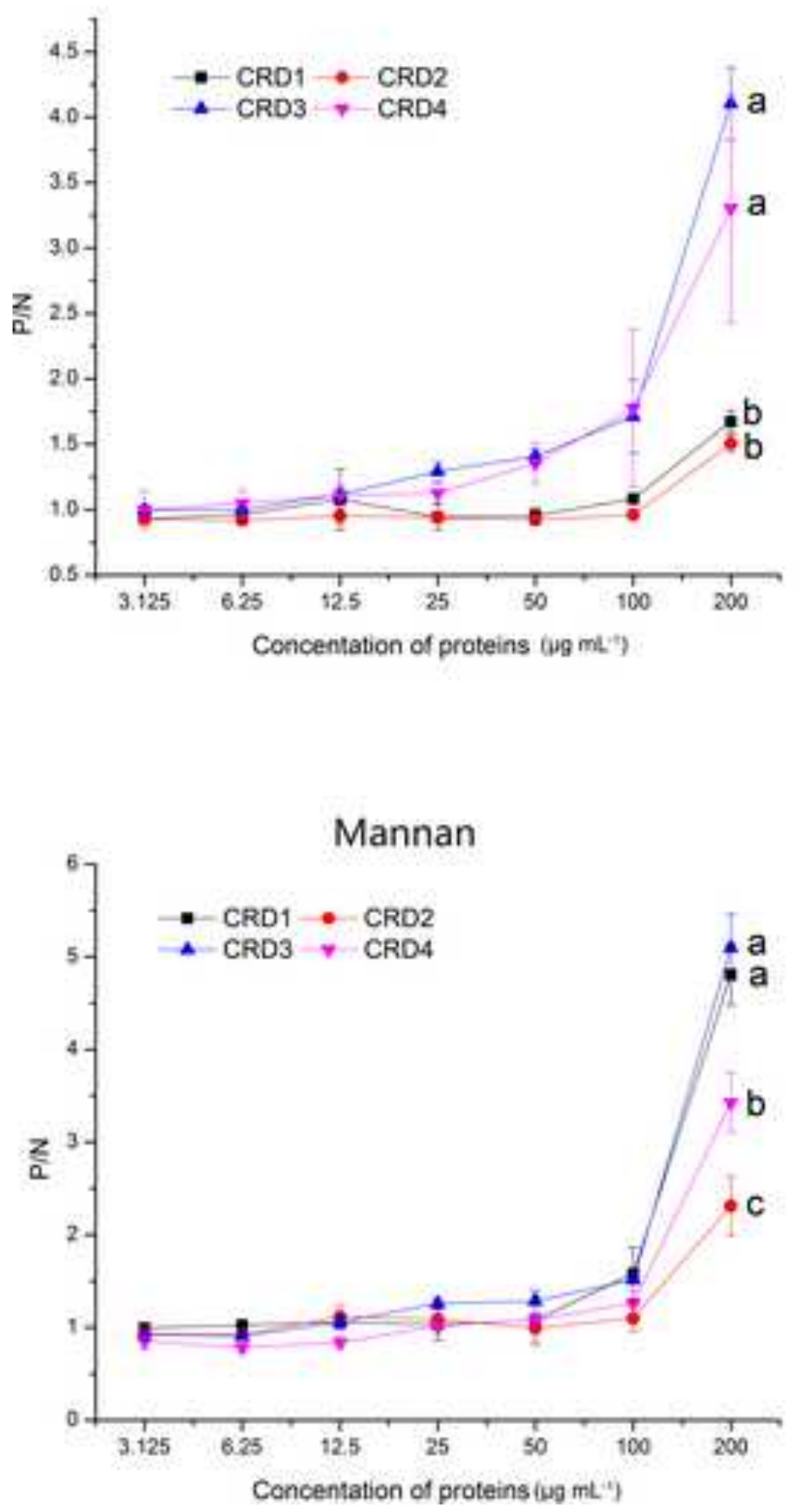

LPS
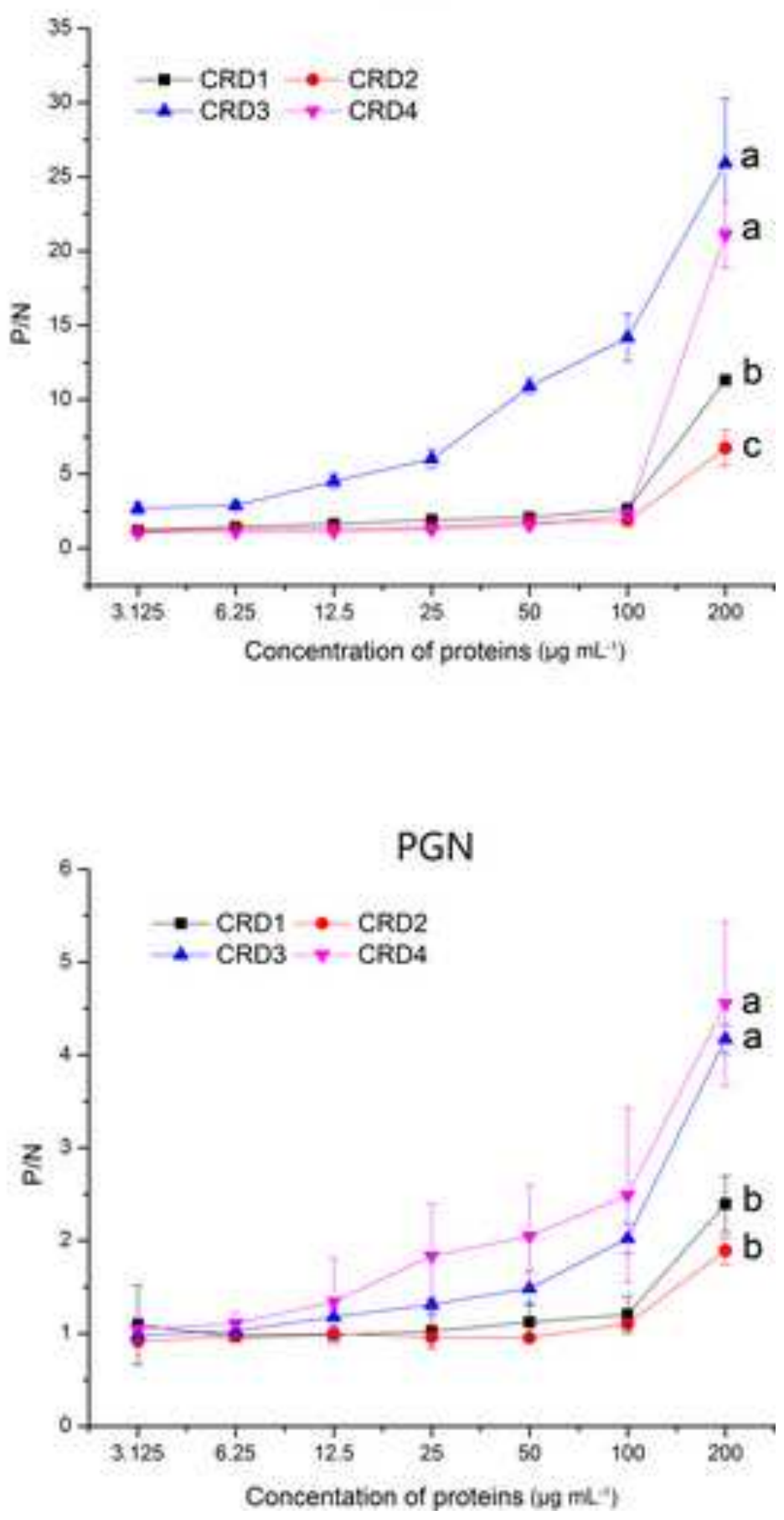


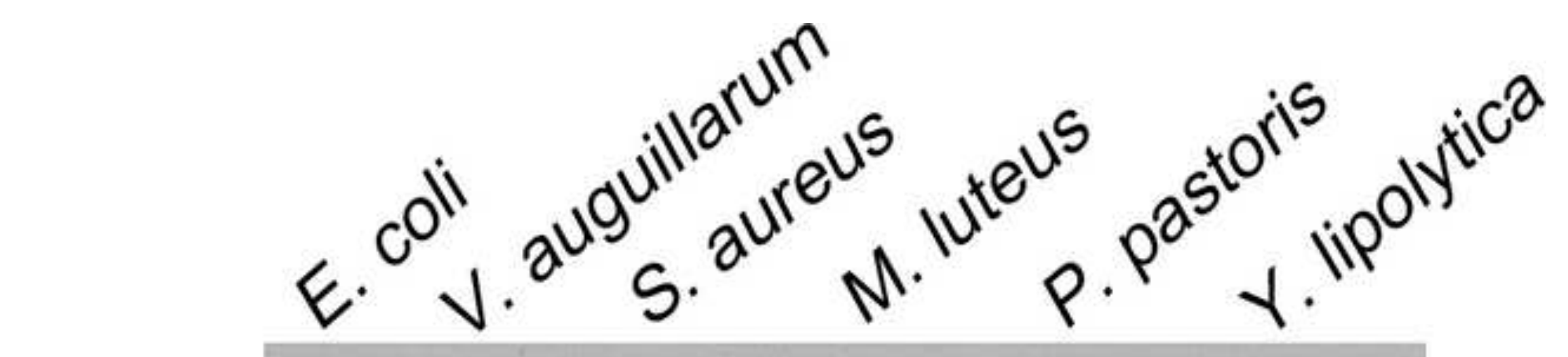

CRD1

CRD2

CRD3
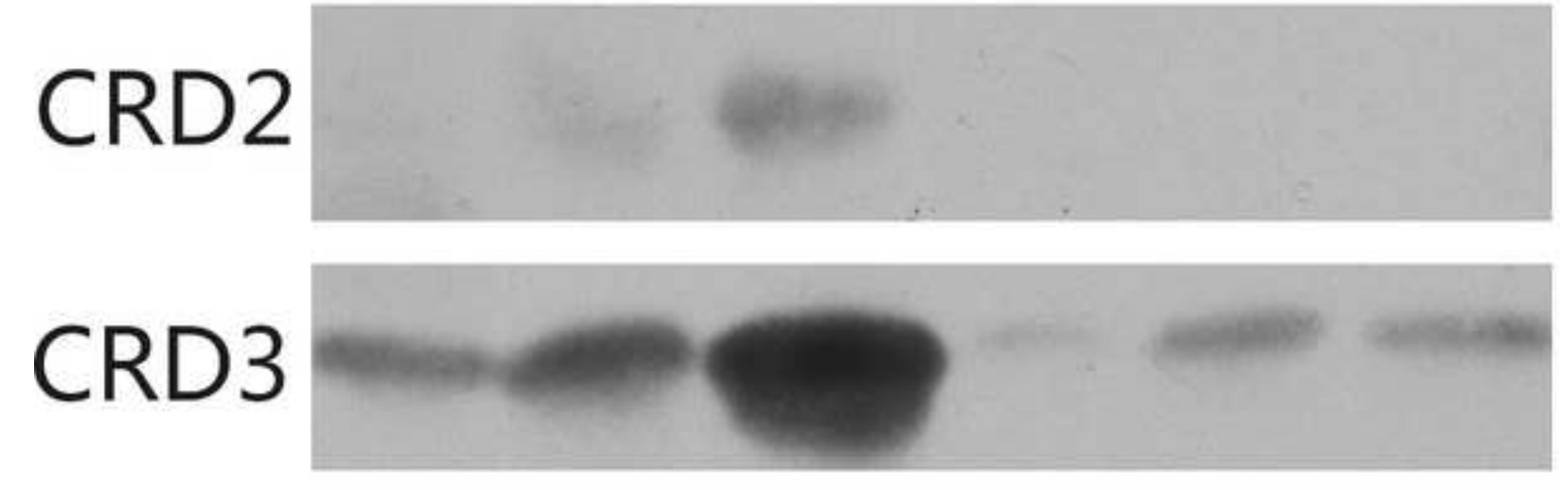

\section{CRD4}




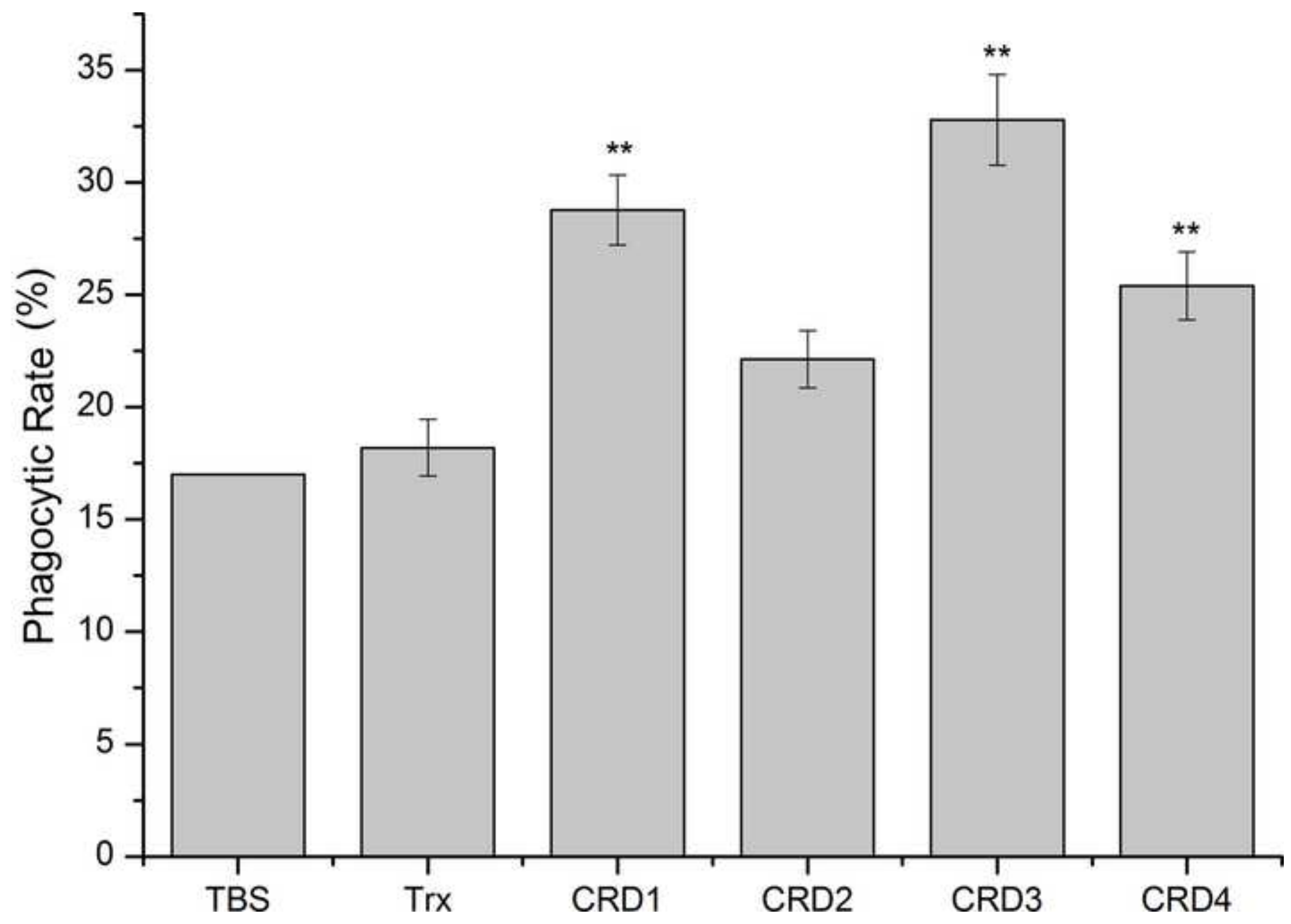

